\title{
24 segment high field permanent sextupole magnets
}

A. Vassiliev, V. Nelyubin, V. Koptev, and P. Kravtsov

High Energy Physics Department, St. Petersburg Nuclear Physics Institute, 188350 Gatchina, Russia

B. Lorentz

Institut für Kernphysik II, Forschungszentrum Jülich, 52425 Jülich, Germany

H. J. Marik

Vacuumschmelze GmbH, P.O.B. 2253, 63412 Hanau, Germany

M. Mikirtytchiants ${ }^{\text {a) }}$ and M. Nekipelov ${ }^{\text {a) }}$

Institut für Kernphysik II, Forschungszentrum Jülich, 52425 Jülich, Germany

F. Rathmann ${ }^{\text {b) }}$

Physikalisches Institut II, Friedrich-Alexander Universität, 91058 Erlangen, Germany

H. Paetz gen. Schieck

Institut für Kernphysik, Universität zu Köln, 50937 Köln, Germany

H. Seyfarth

Institut für Kernphysik II, Forschungszentrum Jülich, 52425 Jülich, Germany

E. Steffens

Physikalisches Institut II, Friedrich-Alexander Universität, 91058 Erlangen, Germany

(Received 25 April 2000; accepted for publication 2 June 2000)

We report on the design, construction, and magnetic field measurements of a system of high field sextupole magnets made from $\mathrm{NdFeB}$ compounds. The magnets are utilized as a focusing system for neutral hydrogen (or deuterium) atoms in a polarized atomic beam source based on Stern-Gerlach spin separation. Each magnet consists of 24 segments of permanently magnetized material differing in remanence and coercivity to reduce demagnetization. According to quadratic extrapolation to the pole tip the magnetic flux density reaches values of up to $B_{0}=1.69 \mathrm{~T}$. Three-dimensional field calculations using the MAFIA code were carried out to optimize the magnet performance and to avoid demagnetization by selecting appropriate materials for the individual segments. Measurements of the radial, azimuthal, and longitudinal magnetic flux density distributions were carried out by means of a small Hall probe $\left(100 \times 200 \times 15 \mu \mathrm{m}^{3}\right)$. The measurements with the small probe permitted to extract experimentally higher order multipole components very close $(\sim 100 \mu \mathrm{m})$ to the inner surface. Experimental values obtained are compared to predictions based on MAFIA calculations and on the Halbach formalism. (C) 2000 American Institute of Physics. [S0034-6748(00)05309-0]

\section{INTRODUCTION}

The production of intense beams of polarized hydrogen and deuterium atoms is of great interest to the nuclear and particle physics community. ${ }^{1}$ Among the various methods employed up to the present time, Stern-Gerlach separation of atoms in inhomogeneous magnetic multipole fields is most widely employed for the production of nuclear spin polarized beams of hydrogen and deuterium. The atomic beam method is applicable to all atoms possessing an unpaired electron in the neutral configuration. ${ }^{2-4}$ In atomic beam sources systems consisting of sextupole magnets are commonly used because of their harmonic focusing properties. The magnitude of the magnetic flux density inside a

\footnotetext{
a) PhD Student from High Energy Physics Department, St. Petersburg Nuclear Physics Institute, 188350 Gatchina, Russia.

b) Author to whom correspondence should be addressed; present address: Institut für Kernphysik II, Forschungszentrum Jülich, 52425 Jülich, Germany; electronic mail: f.rathmann@fz-juelich.de
}

sextupole of inner radius $r_{1}$ and pole-tip field $B_{0}$ at a distance $r$ from the axis of the magnet is given by

$$
B(r)=B_{0}\left(r / r_{1}\right)^{2}
$$

The force acting on atoms with spin projection $m_{j}$ is proportional to the gradient of the magnetic flux density, i.e., proportional to $-m_{j} B_{0} r$. The atoms with $m_{j}=+\frac{1}{2}$ are therefore focused and undergo harmonic motion in $r$ about the magnet axis, while atoms with $m_{j}=-\frac{1}{2}$ are deflected.

The design of multipole magnets consisting of permanently magnetized segments has been described in detail by Halbach. ${ }^{5}$ With the advent of a new generation of magnetic materials in 1984, developed by researchers from Sumitomo, ${ }^{6}$ based on $\mathrm{Nd}$, $\mathrm{Fe}$, and $\mathrm{B}$, energy densities $(B H)_{\max } \sim 290 \mathrm{~kJ} / \mathrm{m}^{3}$ could be achieved, ${ }^{7}$ resulting in a twofold increase over previously existing magnetic materials based on Sm and Co. These new materials were subsequently used in nuclear physics to build magnet systems for ion $^{8-10}$ and atomic beam sources, ${ }^{11-13}$ yielding at most pole- 
TABLE I. Minimal and typical values of the properties of the magnetic materials utilized to manufacture the magnets discussed in this article, remanence $J$, coercivity of polarization $H_{c J}$, and energy density $(B H)_{\max }{ }^{a}$

\begin{tabular}{|c|c|c|c|c|c|c|}
\hline \multirow[b]{2}{*}{ Material } & \multicolumn{2}{|c|}{$\begin{array}{c}\text { Remanence } \\
J(\mathrm{~T})\end{array}$} & \multicolumn{2}{|c|}{$\begin{array}{c}\text { Coercivity } \\
H_{c J}(\mathrm{kA} / \mathrm{m})\end{array}$} & \multicolumn{2}{|c|}{$\begin{array}{l}\text { Energy density } \\
(B H)_{\max }\left(\mathrm{kJ} / \mathrm{m}^{3}\right)\end{array}$} \\
\hline & Min & Typ & Min & Typ & Min & Typ \\
\hline VACODYM 510HR (1) & 1.38 & 1.41 & 950 & 1030 & 360 & 385 \\
\hline VACODYM 383HR (2) & 1.22 & 1.28 & 1430 & 1670 & 275 & 320 \\
\hline VACODYM 400HR (3) & 1.10 & 1.16 & 2150 & 2470 & 225 & 255 \\
\hline
\end{tabular}

${ }^{\mathrm{a}}$ See Ref. 14.

tip fields of $\sim 1.4 \mathrm{~T}$. Modern permanent magnet materials such as VACODYM ${ }^{14} 510 \mathrm{HR}$ reach typical energy densities of $(B H)_{\max } \sim 385 \mathrm{~kJ} / \mathrm{m}^{3}$.

Recently pole-tip fields of 3.2-4.6 T of sextupoles with inner radii of 14-44 $\mathrm{mm}$ have been reported in Ref. 15 using hybrid magnets consisting of superconducting coils surrounded by an iron yoke. A superconducting sextupole system free of iron is described in Ref. 16, which, when excited in a sextupole mode, generates pole-tip fields of $1.54 \mathrm{~T}$ at an inner magnet radius of $65 \mathrm{~mm}$.

In this article we describe the design and construction of permanent sextupole magnets together with detailed field measurements. The work was carried out in the context of the development of a new atomic beam source by an international collaboration. The source is intended for nuclear physics research at the ANKE facility ${ }^{17}$ installed at the Cooler Synchrotron ${ }^{18}$ of the Forschungszentrum Jülich. In Sec. II we describe the design of the sextupole magnets and briefly review the Halbach formalism. Finite-element calculations with the MAFIA code ${ }^{19}$ were carried out on the one hand to optimize the magnetic flux density and on the other to avoid the risk of demagnetization of the material. The final configuration has been assembled from three different magnet materials: VACODYM 510HR, 383HR, and 400HR, recently manufactured by Vacuumschmelze (see Table I). In Sec. III we briefly describe the equipment used to experimentally determine the properties of the magnetic field inside the magnet bore. Section IV contains a summary of the various field measurements of the final magnets. The data are compared to calculations performed with MAFIA and to results obtained from the Halbach formalism.

\section{DESIGN OF SEXTUPOLE MAGNETS}

\section{A. Multipole magnets from permanently magnetized segments}

A cylindrical $2 N$-multipole magnet is assembled from $M$ segments permanently magnetized such that the magnetization axis of each segment advances azimuthally by an angle $(N+1) 2 \pi / M$ with respect to a fixed coordinate system, as sketched in Fig. 1. In Cartesian coordinates using complex numbers, $\mathbf{z}=x+i y=r e^{i \phi}$, the field inside the magnet bore at a distance $r$ from the central axis is given by the Halbach formulas [Ref. 5, Eqs. (24a) and (24b)]

$$
\vec{B}(\mathbf{z})=J \sum_{\nu=0}^{\infty}\left(\frac{\mathbf{z}}{r_{1}}\right)^{n-1} H_{n} K_{n},
$$

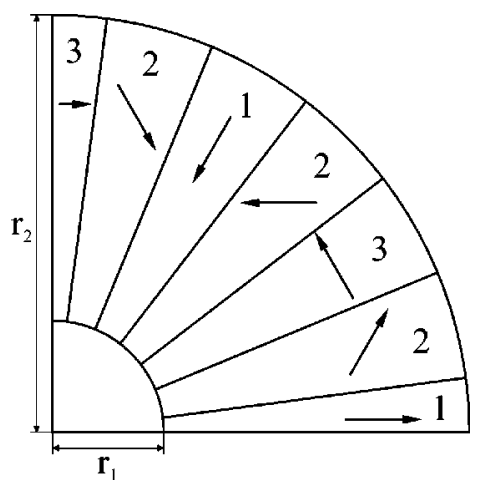

FIG. 1. One quadrant of a cylindrically symmetric sextupole magnet consisting of 24 segments. The arrows denote the direction of magnetization, while the number code indicates the material employed in the final magnet design, listed in Table I.

$$
\begin{aligned}
& n=N+\nu M, \\
& H_{n}=\frac{n}{n-1}\left[1-\left(\frac{r_{1}}{r_{2}}\right)^{n-1}\right], \\
& K_{n}=\frac{\sin [(n+1) \epsilon \pi / M]}{(n+1) \pi / M} .
\end{aligned}
$$

Here the radii $r_{1}$ and $r_{2}$ correspond to the inner and outer boundaries of the magnet, respectively, and $J$ is the remanent magnetization of the material. The filling factor $\epsilon$, which accounts for slits between the segments required for glueing, is close to 1. From the above set of equations [Eqs. (2)-(5)] one can derive the radial and tangential components of the magnetic field as a function of $r$ and $\phi$ in cylindrical coordinates

$$
\begin{aligned}
& B_{\text {rad }}(r, \phi)=J \sum_{\nu=0}^{\infty}\left(\frac{r}{r_{1}}\right)^{n-1} H_{n} \cos (n \phi) K_{n}, \\
& B_{\tan }(r, \phi)=J \sum_{\nu=0}^{\infty}\left(\frac{r}{r_{1}}\right)^{n-1} H_{n} \sin (n \phi) K_{n},
\end{aligned}
$$

where $n, H_{n}$, and $K_{n}$ are defined by Eqs. (3)-(5), respectively. The pole tip field $B_{0}$ of an ideal sextupole magnet given in Eq. (1) is related to quantities in the Halbach formalism presented above by

$$
B_{0}=J_{3} K_{3},
$$

where $\mathrm{H}_{3}$ and $\mathrm{K}_{3}$ are defined by Eqs. (4) and (5), respectively, for $n=3(\nu=0)$.

With respect to the design of sextupole magnets from permanently magnetized material, a number of conclusions can be drawn from the Halbach formalism outlined above:

(i) The pole-tip field of a sextupole magnet, $B_{0}$ [Eq. (8)], consisting of 24 segments exceeds the one of a magnet assembled from 12 segments by $15.5 \%$ (assuming the two magnets compared employ the identical magnet material and are of the same dimensions).

(ii) Deviation from the field generated by an ideal sextupole magnet will manifest itself in higher multipole components only very close to the surface, because the exponent of the radial field dependence for mag- 
TABLE II. Nominal and measured dimensions of the six sextupole magnets, length $\ell$, inner and outer radii $r_{1}$ and $r_{2}$ (Fig. 1), respectively.

\begin{tabular}{cccccccc}
\hline \hline & \multicolumn{3}{c}{ Nominal } & & \multicolumn{4}{c}{ Measured } \\
\cline { 2 - 4 } \cline { 5 - 7 } Magnet & $\begin{array}{c}\ell \\
(\mathrm{mm})\end{array}$ & $\begin{array}{c}r_{1} \\
(\mathrm{~mm})\end{array}$ & $\begin{array}{c}r_{2} \\
(\mathrm{~mm})\end{array}$ & $\begin{array}{c}\ell \\
(\mathrm{mm})\end{array}$ & $\begin{array}{c}r_{1} \\
(\mathrm{~mm})\end{array}$ & $\begin{array}{c}r_{2} \\
(\mathrm{~mm})\end{array}$ \\
\hline 1 & 40 & $5 / 7^{\mathrm{a}}$ & 20 & $40.01(1)$ & $5.20(2) / 7.06(2)^{\mathrm{a}}$ & $19.99(1)$ \\
2 & 65 & $8 / 11^{\mathrm{a}}$ & 32 & $65.01(1)$ & $7.99(1) / 11.06(2)^{\mathrm{a}}$ & $32.02(2)$ \\
3 & 70 & 14 & 47 & $70.01(2)$ & $14.02(1)$ & $47.00(1)$ \\
4 & 38 & 15 & 47 & $38.01(1)$ & $15.02(2)$ & $47.01(1)$ \\
5 & 55 & 15 & 47 & $55.01(1)$ & $15.03(1)$ & $47.00(1)$ \\
6 & 55 & 15 & 47 & $55.00(1)$ & $15.01(2)$ & $47.02(2)$ \\
\hline \hline
\end{tabular}

${ }^{a}$ Magnet with conical opening, the first value denotes the radius at the entrance, the second value the radius of the exit aperture.

nets composed from $M=24$ segments becomes very large, e.g., 26 and 50 for $\nu=1$ and $\nu=2$, respectively [see Eqs. (6) and (7)]. Higher multipole components of the order $\nu=1(n=27)$ and $\nu=2(n=51)$ correspond to azimuthal field dependences of a 54 pole and a 102 pole, respectively.

(iii) For a given inner radius $r_{1}$ of a sextupole magnet the maximum gain in pole-tip field [Eq. (8)] by increasing the outer radius beyond $3 r_{1}\left(4 r_{1}\right)$ is only $12.5(6.7 \%)$.

The magnet design discussed in this article reports for the first time a segmentation into 24 pieces (Fig. 1) even for inner radii down to $r_{1}=5 \mathrm{~mm}$. The focusing properties of the complete magnet system, consisting of six magnets, have been determined from ray tracing calculations using nominal pole-tip fields of $1.5 \mathrm{~T}$. The magnet dimensions, given in Table II, are the result of an optimization procedure with the objective of maximizing the number of atoms transported per unit time into a feeding tube of $10 \mathrm{~mm}$ diameter and $100 \mathrm{~mm}$ length located $300 \mathrm{~mm}$ behind the exit of the last sextupole magnet. The achromatic focusing properties of the magnet system could be improved by making the first two magnets conical, such that the focusing of the magnet system becomes more independent of the particle velocity.

\section{B. Magnet design using MAFIA}

A permanent magnet can be characterized by the remanence $J$ and the (temperature dependent) coercivity of polarization $H_{c J}$. Superposition of the field strengths of different segments leads to demagnetization, if the projection of the resulting field strength on the original magnetization direction is antiparallel and exceeds the maximum coercivity $H_{c J}$ tolerated by a particular segment. Demagnetization presents an unwanted effect because it limits the achievable pole-tip field of the magnets. Therefore, using the nominal dimensions of the magnets (Table II), three-dimensional field calculations with the MAFIA code were carried out to localize these critical regions. A typical result of such a calculation is shown in Fig. 2, where the magnitude of the magnetic flux density $|\vec{B}|$ is shown for one quadrant of magnet 5 in the central plane perpendicular to the longitudinal axis of the magnet. The most critical regions are located near the inner surface of segments labeled $\mathbf{3}$ in Fig. 2, where apparently the magnitude of the magnetic flux density is smallest, hence

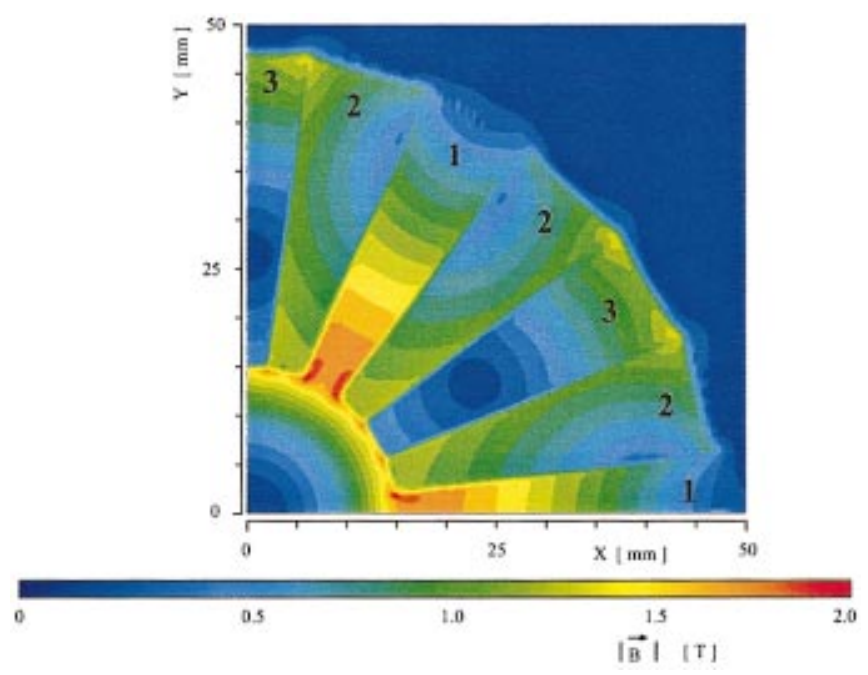

FIG. 2. (Color) Magnitude of the magnetic flux density $|\vec{B}|$ in the central $x y$ plane of sextupole magnet 5 (see Table II for dimensions), calculated using MAFIA. The number code reflects the employed material properties listed in Table I. The original direction of magnetization in the material prior to assembling is indicated in Fig. 1.

according to $\vec{B}=\mu \mu_{0} \vec{H}+\vec{J}$ the field strength $\vec{H}$ is largest and antiparallel to the original magnetization direction. For a more detailed investigation the $x$ component of the field strength $H_{x}$ is plotted in Fig. 3 (left-hand side) as a function of the $y$ coordinate (at $x=0$ ). The critical region is the minimum of $H_{x}$ near the magnet boundary. The resulting field strength $H_{x}=-1.1 \times 10^{3} \mathrm{kA} / \mathrm{m}$ requires to select a material of high coercivity for these segments. The corresponding $x$ component of the magnetic flux density $B_{x}$ is plotted as a function of the $y$ coordinate (at $x=0$ ) in Fig. 3 (right-hand side). As expected, the tangential component of $\vec{H}$ is continuous when passing through the magnet surface, while the tangential component of the magnetic flux density $\vec{B}$ is discontinuous. The $x$ components of $\vec{H}$ and $\vec{B}$ are plotted as a function of the $x$ coordinate (at $y=0$ ) in Fig. 4. The superposition yields a field strength $\vec{H}$ (left-hand side) parallel to the original magnetization direction. Therefore material of highest remanence and lowest coercivity of polarization $(\mathbf{1}$ in Fig. 2) can be employed. The situation for the other segments ( 2 in Fig. 2) is such that medium remanence and medium coercivity of polarization compared to segments $\mathbf{1}$ and 3 are appropriate.

In order to avoid demagnetization, while maximizing the
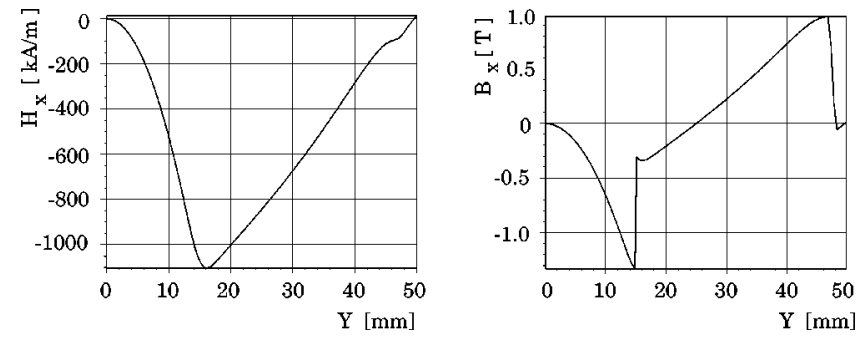

FIG. 3. $x$ component of the magnetic field strength $\vec{H}$ (left-hand side) and magnetic flux density $\vec{B}$ (right-hand side) of magnet 5 (Fig. 2) as a function of the $y$ coordinate at $x=0$. 

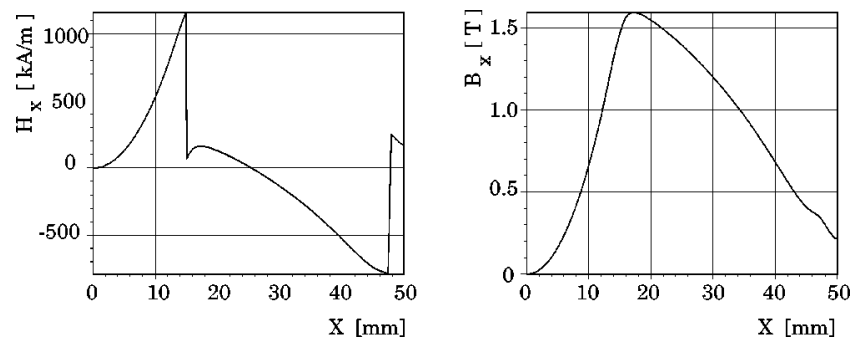

FIG. 4. $x$ component of the magnetic field strength $\vec{H}$ (left-hand side) and magnetic flux density $\vec{B}$ (right-hand side) for magnet 5 as a function of the $x$ coordinate at $y=0$ (Fig. 2).

resulting pole-tip field, an upper limit of the operating temperature of the sextupole magnets around $60^{\circ} \mathrm{C}$ was assumed, which led to the selection of the three magnetic materials employed in the construction of the magnets (Table I). For the segments magnetized in radial direction VACODYM 510HR (1) is used, segments magnetized tangentially are made from VACODYM 400HR (3), for all other segments VACODYM 383HR (2) is employed.

The expected pole-tip field $B_{0}$ of the magnet configuration shown in Fig. 1 was calculated separately for each of the six sextupole magnets from the properties of the employed materials. In the calculation of the expected pole-tip field from the Halbach formalism $B_{0}^{\mathrm{HAL}}$ Eq. (8) is utilized, where only the pure sextupole term of the Halbach series $(\nu=0)$ enters. The results obtained with the minimal and typical values of the average remanence $J$ are given in Table III. The average remanences $J^{\mathrm{min}}=1.233 \mathrm{~T}$ and $J^{\mathrm{typ}}=1.283 \mathrm{~T}$ were calculated taking into account the employed materials (Table I) in proper proportion. The results obtained with MA-

TABLE III. Properties of the sextupole magnets. The range of expected pole-tip fields from the Halbach formalism $B_{0}^{\mathrm{HAL}}$ from Eq. (8), utilizes the average remanence, calculated from the minimal and typical values given in Table I, by taking into account the employed materials in proper proportion (Fig. 1), which yields $J^{\mathrm{min}}=1.233 \mathrm{~T}$ and $J^{\mathrm{typ}}=1.283 \mathrm{~T}$, respectively. The predictions from MAFIA $B_{0}^{\mathrm{MAF}}$ were arrived at by using the typical values of the magnetization of the constituting segments (Table I) and quadratic extrapolation of the resulting flux density to the pole tip of the magnets. Also listed are the measured (extrapolated) pole-tip fields in the central plane at $z=\ell / 2$ of the magnets. $B_{0}$ is obtained from fits using the radial field dependence [Eq. (9)]. $B_{0}^{*}$ is obtained from the azimuthal field dependence using Eqs. (10) and (11). The statistical errors of the fits are indicated in brackets. The uncertainty of the calibration of the Hall probe is $\sim 4 \%$. In the last column we list the radial offset parameter $\Delta r$ obtained from the azimuthal field measurements as well, which denotes the distance between the axis of rotation of the Hall probe and magnet axis.

\begin{tabular}{|c|c|c|c|c|c|c|c|}
\hline \multirow[b]{2}{*}{ Magnet } & \multicolumn{2}{|c|}{$B_{0}^{\mathrm{HAL}}(\mathrm{T})$} & \multicolumn{2}{|c|}{$B_{0}^{\mathrm{MAF}}(\mathrm{T})$} & \multirow[b]{2}{*}{$B_{0}(\mathrm{~T})$} & \multirow[b]{2}{*}{$B_{0}^{*}(\mathrm{~T})$} & \multirow[b]{2}{*}{$\Delta r(\mathrm{~mm})$} \\
\hline & Min & Тур & $\operatorname{Min}^{\mathrm{a}}$ & Тур & & & \\
\hline 1 & 1.607 & 1.672 & 1.569 & 1.633 & $1.654(10)$ & $1.630(4)$ & $0.129(3)$ \\
\hline 2 & 1.615 & 1.680 & 1.577 & 1.641 & $1.684(6)$ & $1.689(2)$ & $0.043(3)$ \\
\hline 3 & 1.609 & 1.674 & 1.578 & 1.642 & $1.625(4)$ & $1.628(4)$ & $0.111(9)$ \\
\hline 4 & 1.586 & 1.650 & 1.503 & 1.564 & $1.565(2)$ & $1.583(3)$ & $0.040(9)$ \\
\hline 5 & 1.586 & 1.650 & 1.542 & 1.605 & $1.621(3)$ & $1.607(2)$ & $0.017(5)$ \\
\hline 6 & 1.586 & 1.650 & 1.542 & 1.605 & $1.621(3)^{b}$ & $1.611(3)$ & $0.176(10)$ \\
\hline
\end{tabular}

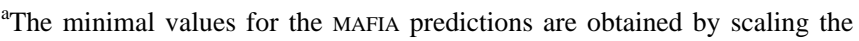
results obtained with the typical values of the average remanence with a factor $J^{\mathrm{min}} / J^{\mathrm{typ}}$.

${ }^{\mathrm{b}}$ The radial dependence of magnet 6 was not separately measured, the value given is for magnet 5 .
FIA were arrived at by using the typical values for the remanence of the individual segments listed in Table I. A quadratic extrapolation to the surface yields the values $B_{0}^{\mathrm{MAF}}$, listed in Table III, where higher order multipole components were suppressed by a proper radial cutoff.

Apparently, the predicted pole-tip fields obtained with MAFIA and from the Halbach formalism, given in Table III, differ systematically. One notices that $B_{0}^{\mathrm{HAL}}$ is always larger than $B_{0}^{\mathrm{MAF}}$ and that the discrepancy between the two calculations is largest for the shortest magnet 4 . This observation can be explained by noting that the Halbach formalism represents a two-dimensional calculation, which assumes that the extension in the third dimension is large, while the MAFIA code represents a true three-dimensional field calculation.

\section{FIELD MAPPING APPARATUS}

The equipment employed during the field mapping of the magnets was chosen such as to allow for:

(i) measurements along the magnet radius,

(ii) full turn azimuthal mapping in the entrance, center, and exit planes of all magnets, and

(iii) mapping parallel to the longitudinal magnet axis.

The measurements were performed using a small Hall probe $\left(200 \times 100 \mu \mathrm{m}^{2}\right.$, thickness $\left.15 \mu \mathrm{m}\right)$ sensitive to the component of the magnetic flux density normal to the probe plane. The probe was mounted on a lathe using a brass support, consisting of a cylindrical centering piece and a narrow finger, sufficiently long to permit measurements along the full longitudinal extension of each of the magnets. The magnets were mounted in tightly fitting aluminum cylinders on the axis of a rotatable table that was attached to the lathe. The positioning accuracy of the probe with respect to the axis of rotation on the lathe was $\sim 10 \mu \mathrm{m}$. The probe plane was carefully adjusted perpendicular with respect to the axis of rotation of the magnets with the short probe edge parallel to the axis. Special care was taken to enable measurements as close as $\sim 100 \mu \mathrm{m}$ from the inner magnet surface in order to access experimentally higher multipole components of the magnetic field. For that purpose it is essential that the active volume of the Hall probe is small, because in a region of a large magnetic field gradient, e.g., near the surface, the Hall probe does not only respond to the radial field components. Corrections arising from the finite size of the Hall probe are discussed in Appendix A.

The probe was calibrated in the range of $0-1.7 \mathrm{~T}$ by comparison to a standard nuclear magnetic resonance (NMR) probe in the homogeneous field of a large dipole magnet. The calibration procedure was repeated after the measurements had been completed. The final calibration curve (magnetic flux density $B$ versus Hall voltage) was obtained from the combined calibration data using a second order polynomial fit. The uncertainty of the Hall probe calibration from the combined calibration data was determined to be $\sim 4 \%$. The temperature coefficient of the employed Hall probe is small $(-0.005 \% / \mathrm{K})$, thus no corrections due to a change in the ambient temperature needed to be applied. The signals 


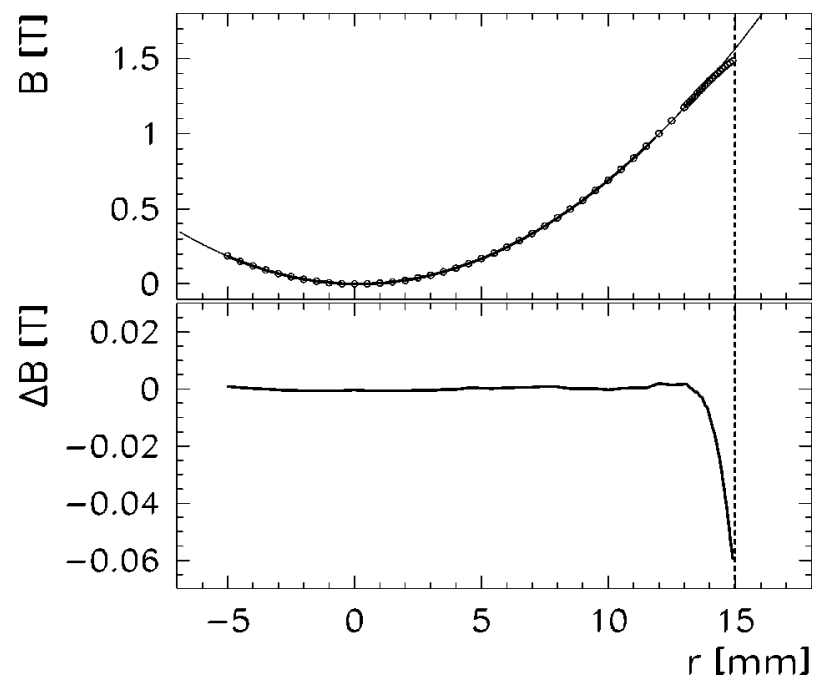

FIG. 5. Magnetic flux density normal to the Hall probe as a function of the radial position for magnet 4 (upper panel). The dashed line indicates the magnet boundary. The solid line represents a fit with Eq. (9), which does not take into account data points at radii above $12 \mathrm{~mm}$. In the lower panel the difference $\Delta B$ between the data and the fit is shown.

from the probe were measured using a multimeter ${ }^{20}$ and read out to a PC via a general purpose interface bias interface.

\section{RESULTS AND COMPARISON TO MAFIA CALCULATIONS}

\section{A. Radial field dependence}

A measurement of the radial magnetic field dependence in the central plane at $\phi=0$ (in front of a pole) yields information about the pole-tip field and about the deviation of magnetic and geometric axis. A typical result of a radial field measurement is shown in Fig. 5. The measured data are fitted with a two parameter function

$$
B(r)=B_{0}\left(\frac{r+\Delta r}{r_{1}}\right)^{2}
$$

where $B_{0}$ is the pole-tip field and $\Delta r$ a radial offset, which accounts for a lateral displacement of the magnet axis with respect to the axis of rotation of the magnet. The radial dependence of the magnetic flux density was measured at least twice for every magnet, once with the magnet at $\phi=0$ and once rotated by $180^{\circ}$. The magnetic field deviates from that of an ideal sextupole in that higher multipole components become sizeable near the magnet surface (see Fig. 5). In the determination of the pole-tip field from a quadratic extrapolation using the resulting parameters of the fit, values obtained at large radii were not taken into account. In Table III the results obtained for $B_{0}$ from fits using Eq. (9) are given for all magnets, except magnet 6 , which is of equal dimension as magnet 5 and was not measured separately. In the extrapolation to the pole tip the nominal magnet dimensions (Table II) were used, except for magnet 1, for which the measured inner radii in the entrance and exit plane differ significantly from the nominal ones. In the listed values for $B_{0}$, the average of the measurements was taken. The displacement $\Delta r$ is on the order of $100 \mu \mathrm{m}$. The errors of $B_{0}$ given in Table III are statistical only.

\section{B. Azimuthal magnetic field dependence}

\section{Medium radii}

Another method for the determination of the pole-tip field of the magnets makes use of the measured azimuthal dependence of the magnetic fields. For that purpose the magnets were rotated in steps of $4^{\circ}$. Measurements were taken at different radii and in planes perpendicular to the axis of rotation at the entrance, center, and exit of the magnet. In order to reduce the influence of higher multipole components, measurements at medium radii were selected, for which the contribution to the measured magnetic flux density from amplitudes of higher harmonics $(\nu=1, \ldots, \infty)$ is at most 3 $\times 10^{-3}$ of the total amplitude given in Eqs. (2)-(5). In this case the pole-tip field can be extracted from a fit to the azimuthal field dependence. As in the measurements of the radial field dependence discussed above, a correction needs to be applied, which takes into account a lateral displacement $\Delta r$ between the axis of rotation of the table and that of the magnet. The displacement, $\Delta r \sim 100 \mu \mathrm{m} \ll r_{1}$, is mainly caused by mechanical inaccuracies due to the protective measure to mount the magnets inside aluminum cylinders on the rotatable table of the lathe. The fitting function for the magnetic flux density as a function of radius $r$ and azimuth $\phi$ for the component normal to the Hall probe is given by

$$
B_{\text {norm }}(r, \phi)=J \sum_{\nu=0}^{\infty}\left(\frac{r}{r_{1}}\right)^{n-1} H_{n} K_{n} C_{n} .
$$

The correction factor

$$
\begin{aligned}
C_{n}= & {\left[1+(n-1) \frac{\Delta r}{r} \cos \left(\phi-\phi_{0}^{\Delta r}\right)\right] } \\
& \cdot \cos \left[n \phi+\phi_{0}^{r}-(n+1) \frac{\Delta r}{r} \sin \left(\phi-\phi_{0}^{\Delta r}\right)\right],
\end{aligned}
$$

introduced in Eq. (10), is derived in Appendix B. The angles $\phi_{0}^{r}$ and $\phi_{0}^{\Delta r}$ correspond to the initial phases. One example of a measured azimuthal field dependence is depicted in Fig. 6. The resulting pole-tip field values $B_{0}^{*}$ obtained from this procedure are listed in Table III. The two different methods give consistent results.

\section{Large radii}

Measurements at large radii (near the surface) yield information about higher multipole components. One example of such a measurement on magnet 5 at a radius of $r=14.9$ $\mathrm{mm}$ with a step of $4^{\circ}$ is shown in Fig. 7. At this radius, the amplitudes of the higher harmonics $\nu=1(\nu=2)$ according to the Halbach formulas [Eqs. (2) $-(5)]$ correspond to $10.0 \%$ $(4.5 \%)$ of the total amplitude. Additional azimuthal measurements were carried out with a smaller step of $0.5^{\circ}$ for each of the six sextupole magnets discussed here in a reduced angular range (one quadrant). In Fig. 8 the azimuthal field dependence is shown for magnet 5 . The results of a MAFIA calculation are also shown in Fig. 8. The agreement of the measured data with the MAFIA calculation is quite good. A fitted curve using Eqs. (10) and (11) for $\nu=0, \ldots, 10$ is also indicated. In the fitting procedure the amplitudes of the multipole components have to be corrected by taking into ac- 


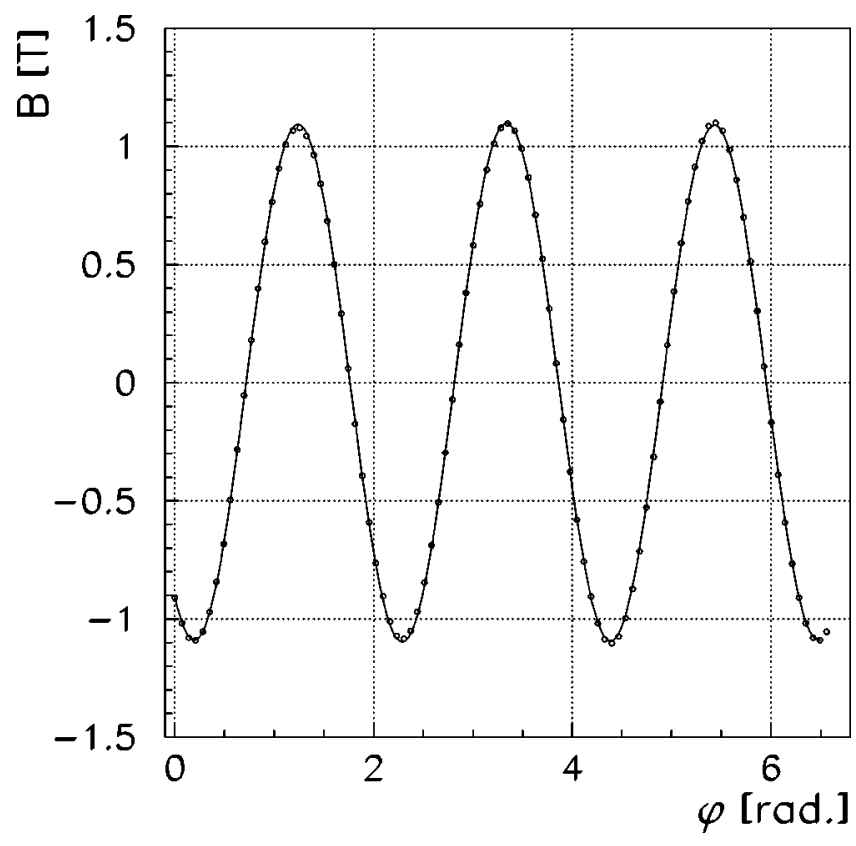

FIG. 6. Azimuthal dependence of $B_{\text {rad }}$ for magnet 4 at a radius of $12.5 \mathrm{~mm}$. The solid line represents a fit with a function taking into account the field component normal to the Hall probe and a radial offset [Eqs. (10) and (11)].

count the finite size of the Hall probe. This is discussed in detail in Appendix A. (The correction terms for a Hall probe of $h=0.2 \mathrm{~mm}$ lateral dimension are listed in Table IV.) From the fit an average remanence of $J=1.293 \pm 0.008 \mathrm{~T}$ results, which agrees well with the expected value for the average remanence $J^{\text {typ }}=1.283 \mathrm{~T}$, calculated from the employed materials. Also shown in Fig. 8 is the $\nu=0$ term obtained from the fit with $\nu=0, \ldots, 10$, which corresponds to a pure sextupole. Omitting higher order terms $\nu>10$ is justified, because the amplitude of all higher harmonics corresponds to only about $1 \%$ of the total amplitude.

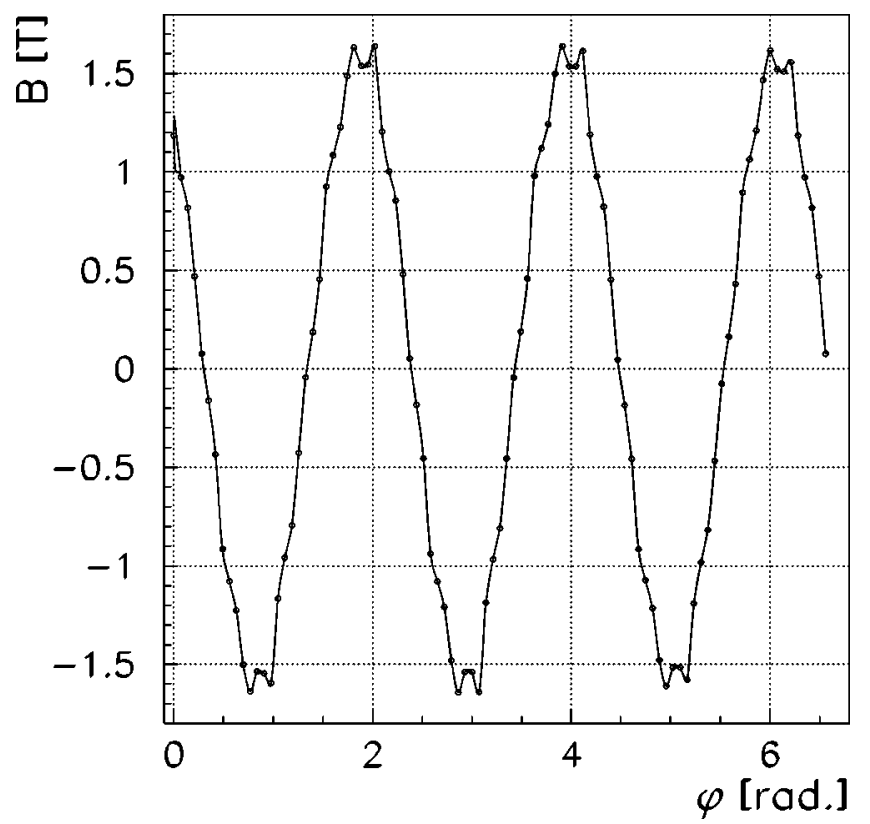

FIG. 7. Azimuthal dependence of $B_{\text {rad }}$ for magnet 5 at a radius of $14.9 \mathrm{~mm}$. (The solid line serves to guide the eye.)

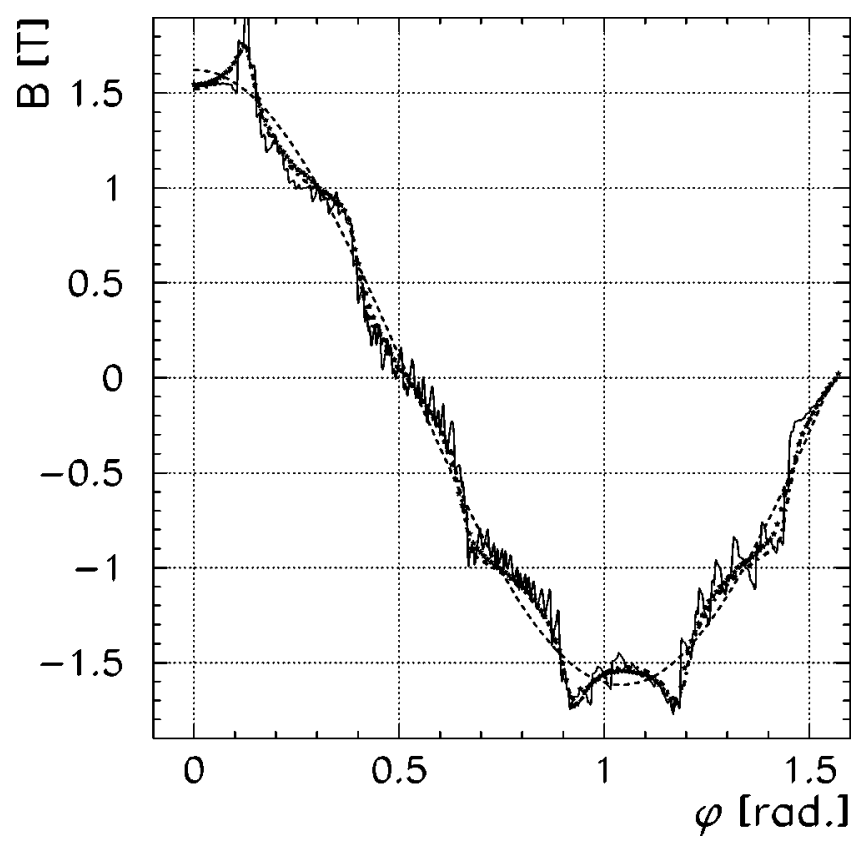

FIG. 8. Azimuthal dependence of $B_{\text {rad }}$ for magnet 5 at a radius of $14.9 \mathrm{~mm}$ for one quadrant. The measured data $(\star)$ are shown together with the results of a MAFIA calculation (thin solid line). A fit to the data with Eqs. (10) and (11) for $\nu=0, \ldots, 10$ of the Halbach series using as a free parameter an average remanence $J$ is indicated (dash-dotted) and yields $J=1.293$ $\pm 0.008 \mathrm{~T}$, in good agreement with the expectation $J^{\mathrm{typ}}=1.283 \mathrm{~T}$. The multipole amplitudes in the fit were corrected as described in Appendix A, in order to account for the finite size of the Hall probe. The $\nu=0$ term of that fit alone (pure sextupole) is indicated as well (dashed line).

In order to access the first order multipole component $\nu=1$ the difference between a pure sextupole $\nu=0$ and the measured data, both shown in Fig. 8, is computed and plotted in Fig. 9. The resulting distribution exhibits the typical frequency of the next harmonic $\nu=1$, which corresponds to a 54 pole. Also plotted in this graph is the result of the Halbach fit to the azimuthal distribution shown in Fig. 8 for $\nu$ $=1, \ldots, 10$. Subtraction of both terms, $\nu=0$ and $\nu=1$ of the fitted Halbach series from the data, yields the distribution shown in Fig. 10. The typical 102-pole structure of the $\nu$ $=2$ term is clearly visible.

A measurement of the azimuthal field dependence at the entrance and exit of each magnet was carried out in order to

TABLE IV. Correction terms $C(\nu)$ calculated for magnet 1 (inner radius $r_{1}=6 \mathrm{~mm}$ ) at $r=5.9 \mathrm{~mm}$ and for a magnet with an inner radius of $r_{1}=15$ $\mathrm{mm}$ at $r=14.9 \mathrm{~mm}$ up to order $\nu=10$ for a Hall probe of $h=0.2 \mathrm{~mm}$.

\begin{tabular}{rcc}
\hline \hline & $\begin{array}{c}C \nu, r_{1}=6 \mathrm{~mm}, \\
\nu\end{array}$ & $\begin{array}{c}C\left(r_{1}=15 \mathrm{~mm},\right. \\
r=5.9 \mathrm{~mm}, h=0.2 \mathrm{~mm})\end{array}$ \\
\hline 0 & 0.999 & $14.9 \mathrm{~mm}, h=0.2 \mathrm{~mm})$ \\
1 & 0.964 & 1.000 \\
2 & 0.877 & 0.994 \\
3 & 0.747 & 0.980 \\
4 & 0.587 & 0.958 \\
5 & 0.410 & 0.927 \\
6 & 0.234 & 0.889 \\
7 & 0.073 & 0.844 \\
8 & -0.060 & 0.793 \\
9 & -0.156 & 0.736 \\
10 & -0.211 & 0.675 \\
\hline \hline
\end{tabular}




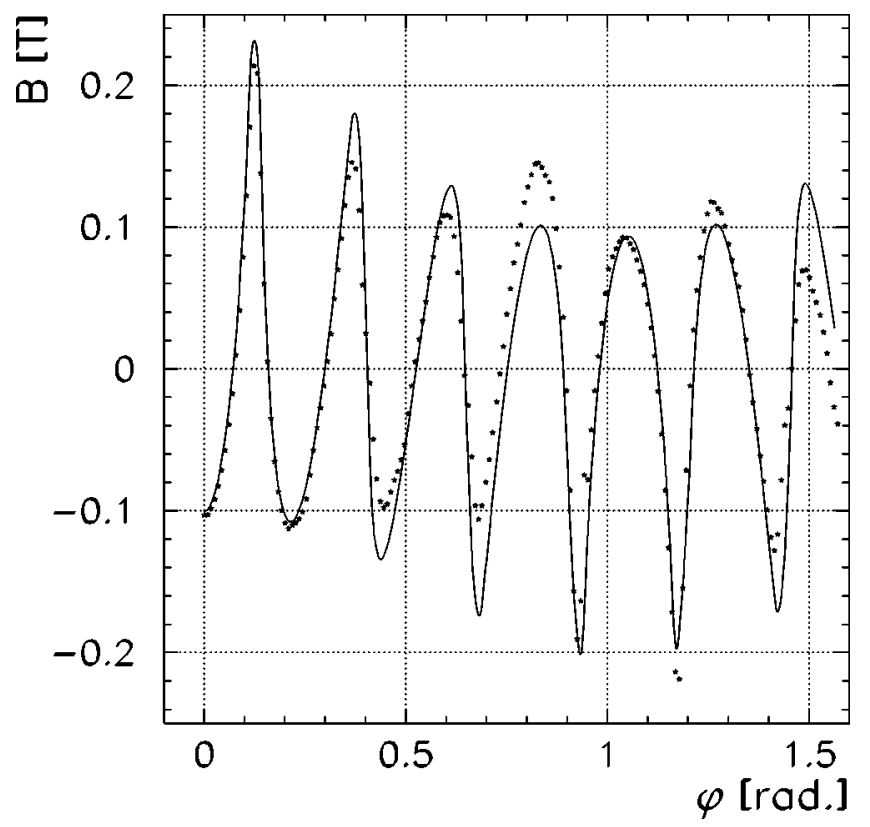

FIG. 9. Multipole components $\nu \geqslant 1(\star)$, corresponding mainly to a 54 pole $(\nu=1)$, extracted from the measured data shown in Fig. 8 by subtraction of the pure sextupole component $\nu=0$ from the data. The Halbach series for $\nu=1, \ldots, 10$ of the fit to the data of Fig. 8 with the full series ( $\nu$ $=0, \ldots, 10)$ is indicated as a solid line.

detect a possible spirality of the magnets. It was found that the positions of the maxima of the magnetic flux density in the entrance and exit plane coincide within $1^{\circ}$ with the position of the maxima found in the center plane.

\section{Small radii}

Measurements at small radii were carried out as well. While at medium and large radii the effects of a nonpoint-

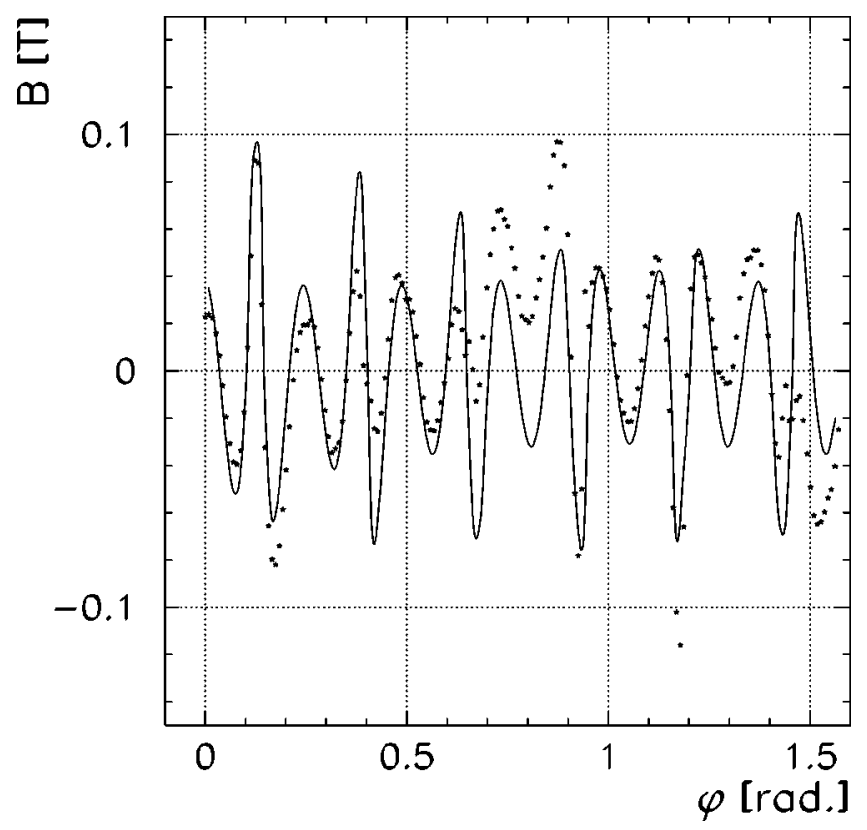

FIG. 10. Multipole components $\nu \geqslant 2(\star)$ extracted from the data by subtraction of $\nu=0$ and $\nu=1$ terms from the data shown in Fig. 8 yield the typical 102-pole structure, corresponding to $\nu=2$. Also shown as a solid line is the result of the fit with the Halbach series $(\nu=0, \ldots, 10$, shown in Fig. 8) for $\nu=2, \ldots, 10$ only.

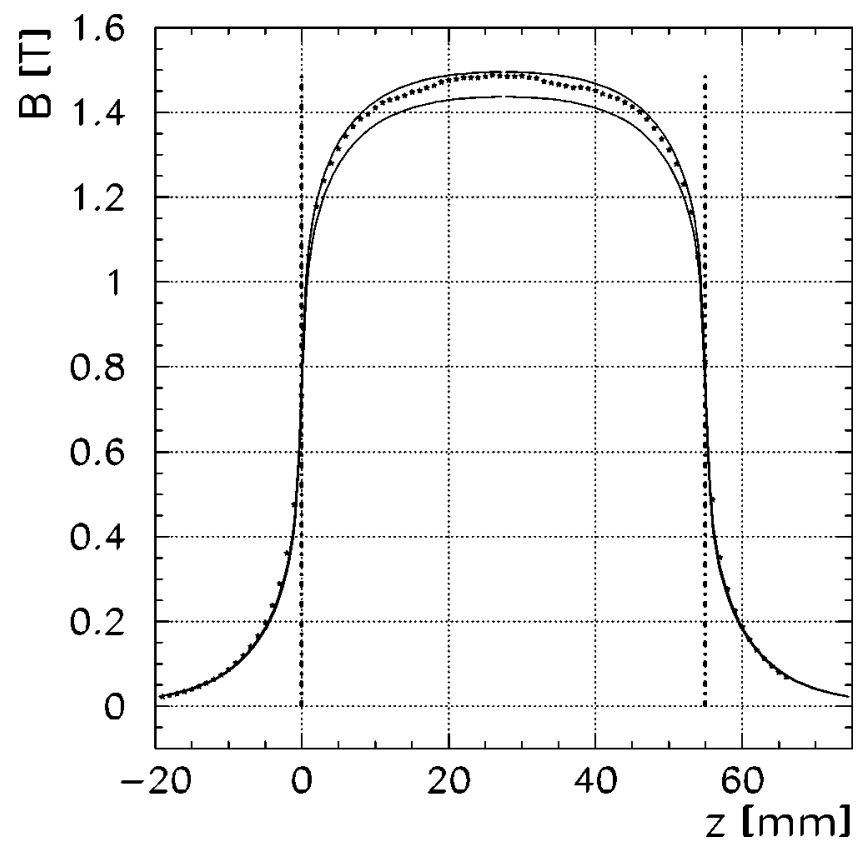

FIG. 11. Magnetic flux density $B_{\text {rad }}(\star)$ along the axis of magnet 6 at a radius of $14.5 \mathrm{~mm}$ at $\phi=0$. The upper solid line indicates the result of a MAFIA calculation using typical values for the remanence $J^{\text {typ }}$ of the individual segments (Table I). The lower solid line for the configuration with minimal values of the magnetization is obtained by scaling the results obtained with $J^{\text {typ }}$ by a factor $J^{\mathrm{min}} / J^{\text {typ }}$. The dashed lines correspond to the physical boundaries of the magnet.

like Hall probe can either be ignored or corrected for, at small radii these effects become large and make a meaningful measurement virtually impossible. A detailed discussion of finite-size Hall probe effects is given in Appendix A.

\section{C. $\boldsymbol{z}$ dependence}

In order to investigate the uniformity of the magnets, measurements of $B_{\text {rad }}$ in an axial direction were carried out. One such measurement on magnet 6 (cylindrical bore) at a radius of $14.5 \mathrm{~mm}$ is shown in Fig. 11. The agreement between the data and the results of a MAFIA calculation, also shown in the figure, is good. In Fig. 12 the result of a measurement of $B_{\text {rad }}$ parallel to the axis of magnet 1 (conical bore) at a radius of $4.5 \mathrm{~mm}$ is shown. The solid line corresponds to a calculation based on the measured pole-tip field $B_{0}^{*}$ (Table III) and the Halbach formulas, in that for each longitudinal coordinate an infinitely long magnet with the appropriate inner and outer radii, $r_{1}$ and $r_{2}$, respectively, is assumed.

Ray tracing calculations in most cases assume a field integral $\int B_{\text {rad }}(z) d z$ calculated for a box-shaped magnetic flux density, as indicated in Fig. 12. In Table V we compare the calculated field integrals of the six sextupole magnets discussed here to the measured ones, obtained by integration over the measured $z$ dependence. The calculated values for the cylindrical magnets were obtained using the pole-tip field values $B_{0}^{*}$, listed in Table III, multiplied by a factor $\left(r / r_{1}\right)^{2}$ to account for the radius at which the longitudinal field dependence was measured. For the conical magnets the measured pole-tip field in the center plane, $B_{0}^{*}$, was used also. Here in addition to the factor discussed above, the change in 


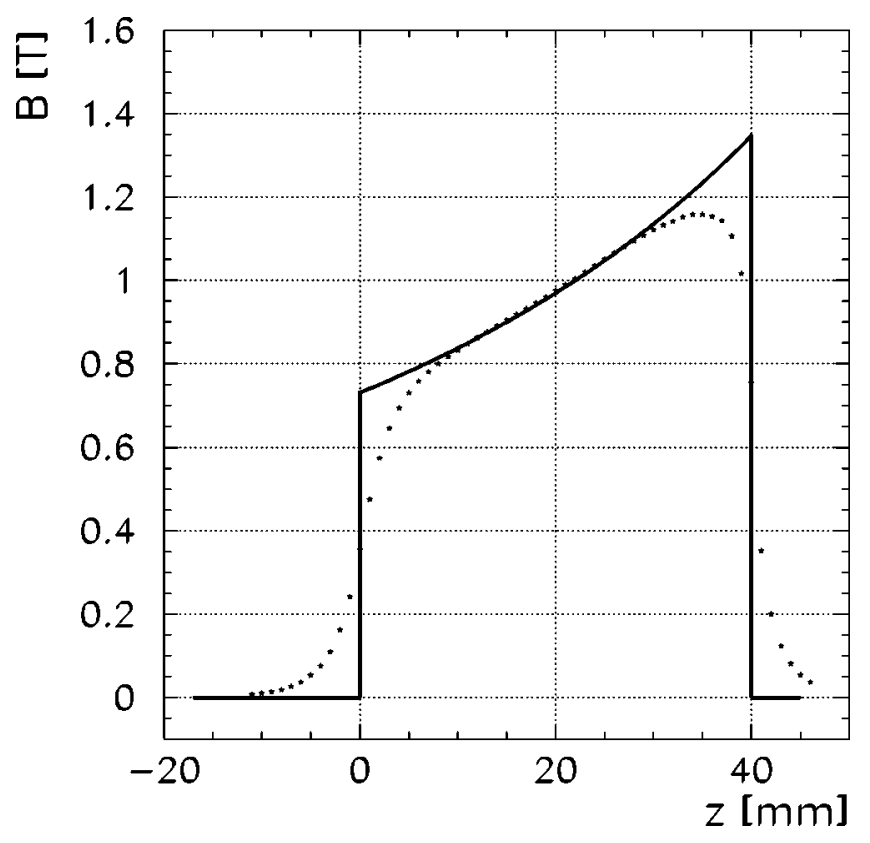

FIG. 12. Measured magnetic flux density $B_{\text {rad }}$ along the axis of magnet 1 $(\star)$ at a radius of $4.5 \mathrm{~mm}$ at $\phi=0$. The solid line corresponds to the result of a calculation based on the measured pole-tip field $B_{0}^{*}$ (Table III), using a factor $\left(r / r_{1}\right)^{2} \cdot\left[1-\left(r_{1} / r_{2}\right)^{2}\right]$ to account for the radius $r$ at which the distribution was measured and for the change in material thickness.

magnet thickness was factored in using $\left[1-\left(r_{1} / r_{2}\right)^{2}\right]$. Except for magnet 1 , where the discrepancy between measured and calculated field integral reaches $\sim 10 \%$, the agreement is quite good (Table V).

\section{DISCUSSION}

We have reported on the development of segmented sextupole magnets which employ a new generation of permanently magnetized materials now available. The six permanent magnets consist of 24 segments with inner radii down to $5 \mathrm{~mm}$. This represents an improvement because previously for radii of that size only magnets consisting of 12 segments have been produced. ${ }^{13}$ It should be pointed out that the substantial increase in pole-tip field of about $16 \%$ going from 12 to 24 segments reduces to a mere $3.5 \%$ when the number of segments is increased by another factor of 2 to 48 segments. Thus the additional technical effort involved in building 48segmented magnets is presumably not justified.

The MAFIA code is a powerful tool that can be used efficiently to predict with good accuracy the pole-tip field

TABLE V. Measured and calculated values for the field integrals $\int B_{\mathrm{rad}}(z) d z$ of the six magnets at radii $r$.

\begin{tabular}{|c|c|c|c|}
\hline Magnet & $r(\mathrm{~mm})$ & $\begin{array}{c}\text { Measured } \\
\int B_{\mathrm{rad}}(z) d z(\mathrm{~T} \mathrm{~mm})\end{array}$ & $\begin{array}{c}\text { Calculated } \\
\int B_{\mathrm{rad}}(z) d z(\mathrm{~T} \mathrm{~mm})\end{array}$ \\
\hline 1 & 4.5 & 40.11 & 36.05 \\
\hline 2 & 7.5 & 71.12 & 70.18 \\
\hline 3 & 13.5 & 101.92 & 105.77 \\
\hline 4 & 14.5 & 57.68 & 55.57 \\
\hline 5 & 14.5 & 83.47 & 83.31 \\
\hline \multirow[t]{2}{*}{6} & 7.5 & 22.33 & 22.28 \\
\hline & 14.5 & 84.15 & 83.31 \\
\hline
\end{tabular}

and all other observed features of segmented multipole magnets. The only uncertainty in the prediction arises from the insufficient knowledge of the remanence of the materials employed, which holds for the Halbach formulas as well.

The measurements presented here constitute the first experimental determination of amplitudes and multipolarities related to the first and second order $(\nu=1,2)$ multipole field components ever carried out for segmented multipole magnets. We have confirmed experimentally, that the Halbach formalism ${ }^{5}$ for permanent multipole magnets consisting of a finite number of segments of different magnetization describes these tiny effects near the magnet surface with good accuracy, in particular in the azimuthal field dependences.

We find no experimental evidence for the statements made in Ref. 11 with respect to the multipolarity in sextupole magnets consisting of $M=24$ segments, namely that the expected higher order symmetries correspond to an 18 pole and a 30 pole.

Since higher multipole components appear only very close to the magnet surface, the number of atoms affected by them is small. In addition, it can be shown using the Halbach formulas that the integrals over the full azimuth of the gradient of the magnitude of the magnetic flux density for a pure sextupole and for the more realistic case including higher multipole components (e.g., $\nu=0, \ldots, 100)$ yield the same results. Thus one can conclude that higher multipole components in a segmented sextupole magnet have no effect on the magnets' focusing properties.

One of the conditions for the validity of the Halbach formalism is the spatial independence of the magnetization vector $\mathbf{J}$ within each magnet segment. It is treated as a constant scalar $J$. Rather inhomogeneous magnet material would result in the appearance of more and other harmonics. The fact that we found exactly the harmonics predicted by Halbach proves the good homogeneity of the magnet material used.

The pole-tip field values for the six magnets reported here, with values in the range of $1.58-1.69 \mathrm{~T}$, represent a substantial improvement for the magnet systems employed in atomic beam sources. The larger pole-tip field enables the use of wider magnet apertures, without compromising the field gradient. Since the acceptance of a magnet system is thereby increased, a somewhat higher flow of polarized atoms transported by the magnet system should be obtained.

\section{ACKNOWLEDGMENTS}

The authors wish to thank Dr. V. Pogodin from the Ioffe Physico-Technical Institute, 194021 St. Petersburg, Russia, for providing the Hall probe, and V. Trofimov for building the power supply of the Hall probe and for his help during the NMR calibration measurements. They gratefully acknowledge the help of the mechanical workshop of the Institut für Kernphysik of the Forschungszentrum Jülich during the measurements. This work has been supported by the BMBF under Contract Nos. RUS-649-96 and 06 ER 831, by the Forschungszentrum Jülich (FFE) under Contract No. 


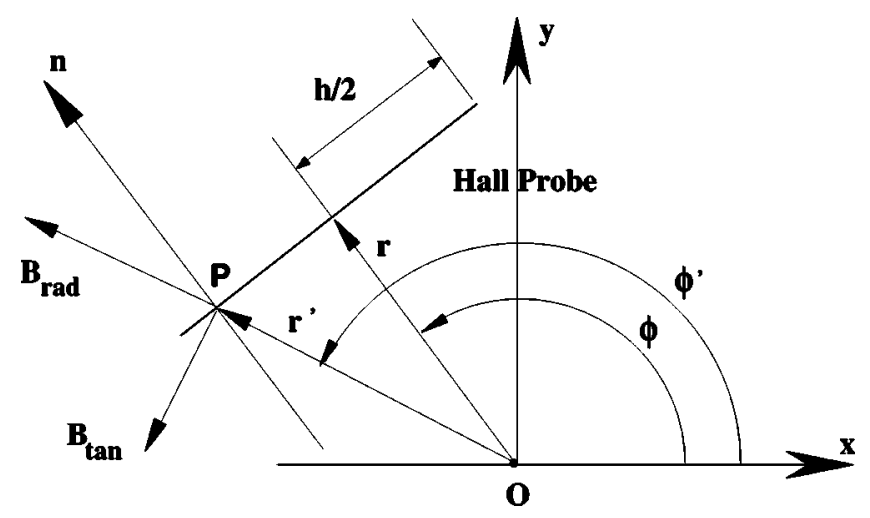

FIG. 13. Coordinate system to determine the influence of the finite size of the lateral dimension $h$ of the Hall probe.

41149451, by the Deutsche Forschungsgemeinschaft under Contract No. 436 RUS 113/430, and by the Russian Ministry of Sciences.

\section{APPENDIX A: HALL PROBE FINITE SIZE EFFECTS}

The magnitude and direction of the magnetic flux density inside a multipole magnet change within the volume of the Hall probe that is used to measure the field. Therefore, it is necessary to investigate to what extent the finite size of the detector probe has to be taken into account. The discussion presented here refers to measurements carried out with a Hall probe, where the sensitive plane is perpendicular to the radius of the magnet. The signal measured by the Hall probe is proportional to the normal component of the magnetic flux density $B_{\text {norm }}$ averaged over the lateral dimension $h$ of the probe, as indicated in Fig. 13:

$$
B_{\text {meas }}=\frac{1}{h} \cdot \int_{-h / 2}^{h / 2} B_{\text {norm }} d l .
$$

The normal component at point $P$ [coordinates $\left(r^{\prime}, \phi^{\prime}\right)$ in Fig. 13] is given by

$$
\begin{aligned}
B_{\text {norm }}\left(r, \phi, r^{\prime}, \phi^{\prime}\right)= & B_{\text {rad }}\left(r^{\prime}, \phi^{\prime}\right) \cdot \cos \left(\phi^{\prime}-\phi\right) \\
& -B_{\text {tan }}\left(r^{\prime}, \phi^{\prime}\right) \cdot \sin \left(\phi^{\prime}-\phi\right) .
\end{aligned}
$$

$B_{\text {rad }}$ and $B_{\tan }$ are defined in Eqs. (6) and (7), where $r$ and $\phi$ are the coordinates of the center of the Hall probe. The above expression converts into

$$
\begin{aligned}
B_{\text {norm }}\left(r, \phi, \phi^{\prime}\right)= & J \cdot \sum_{\nu=0}^{\infty} H_{n} K_{n} \\
& \cdot\left(\frac{r}{r_{1}}\right)^{n-1} \frac{\cos \left((n+1) \phi^{\prime}-\phi+\phi_{0}^{r}\right)}{\cos ^{n-1}\left(\phi^{\prime}-\phi\right)},
\end{aligned}
$$

where $\phi_{0}^{r}$ denotes the starting phase. Integration of Eq. (A1) using for $B_{\text {norm }}$ the expression from Eq. (A3) yields

$$
B_{\text {meas }}=J \cdot \sum_{\nu=0}^{\infty} H_{n} K_{n} \cdot\left(\frac{r}{r_{1}}\right)^{n-1} \cos \left(n \phi+\phi_{0}^{r}\right) \cdot C(\nu, h),
$$

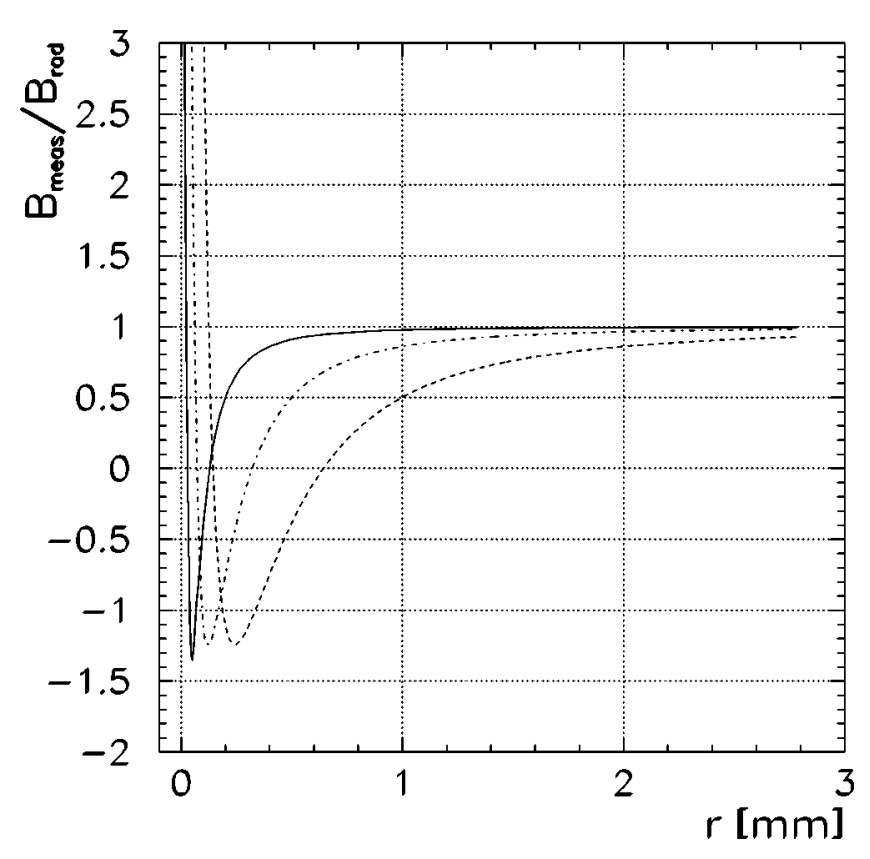

FIG. 14. Calculated ratio of magnetic flux densities $B_{\text {meas }} / B_{\text {rad }}$ near the magnet axis for three different Hall probe dimensions. The solid line corresponds to $h=0.2 \mathrm{~mm}$, the dashed-dotted line to $h=0.5 \mathrm{~mm}$, and the dashed line to $h=1.0 \mathrm{~mm}$.

where the effects of a nonpoint-like Hall probe have been taken into account by a correction term $C(\nu, h)$ that depends on the order $\nu$ of the harmonic contributions involved and on the lateral dimension $h$ of the Hall probe employed in the measurements. The correction term is given by

$$
C(\nu, h)=\left(\frac{2 r}{h}\right) \cdot \int_{0}^{\arctan (h / 2 r)} \frac{\cos [(n+1) \xi]}{\cos ^{n+1} \xi} d \xi .
$$

It should be noted that $C(\nu, h)$ is independent of the azimuth $\phi$ and that for an ideal (point-like) Hall probe $C(\nu, h=0)$ $\equiv 1$. Although it is possible to express the integral given above through elementary functions, here we discuss the effect of the correction term in the central plane for two of our magnets: the smallest one with $r_{1}=6 \mathrm{~mm}$ and a magnet with $r_{1}=15 \mathrm{~mm}$. In Table IV the correction factors are listed that have to be applied to the multipole components up to order $\nu=10$ in a measurement where the Hall probe is positioned at $r=14.9 \mathrm{~mm}\left(r_{1}=15 \mathrm{~mm}\right)$ and at $r=5.9 \mathrm{~mm}\left(r_{1}=6 \mathrm{~mm}\right)$, respectively. For $\nu=2$, for the small (large) aperture magnet with a probe of lateral dimension $h=0.2 \mathrm{~mm}$ the correction is already sizeable, $\sim 12.3 \%(\sim 2.0 \%)$. For larger Hall probe dimensions, e.g., $h=0.5 \mathrm{~mm}$, the correction term for the small aperture magnet for $\nu=2$ amounts to $\sim 63.5 \%$. Thus it is not feasible to study effects related to higher order multipolarities with Hall probes of large lateral dimension.

At small radii $(r<3 \mathrm{~mm})$, multipole components other than $\nu=0$ can be neglected. However, the corrections arising from the nonpoint-like Hall probe become large as well, as indicated in Fig. 14, where the ratio of the ideal (point-like) and measured magnetic flux density $B_{\text {meas }} / B_{\text {rad }}$ is plotted for radii $r$ below $3 \mathrm{~mm}$ for the three lateral probe dimensions $(h=0.2,0.5$, and $1.0 \mathrm{~mm})$. One must conclude that it is not possible to carry out meaningful measurements at small radii 


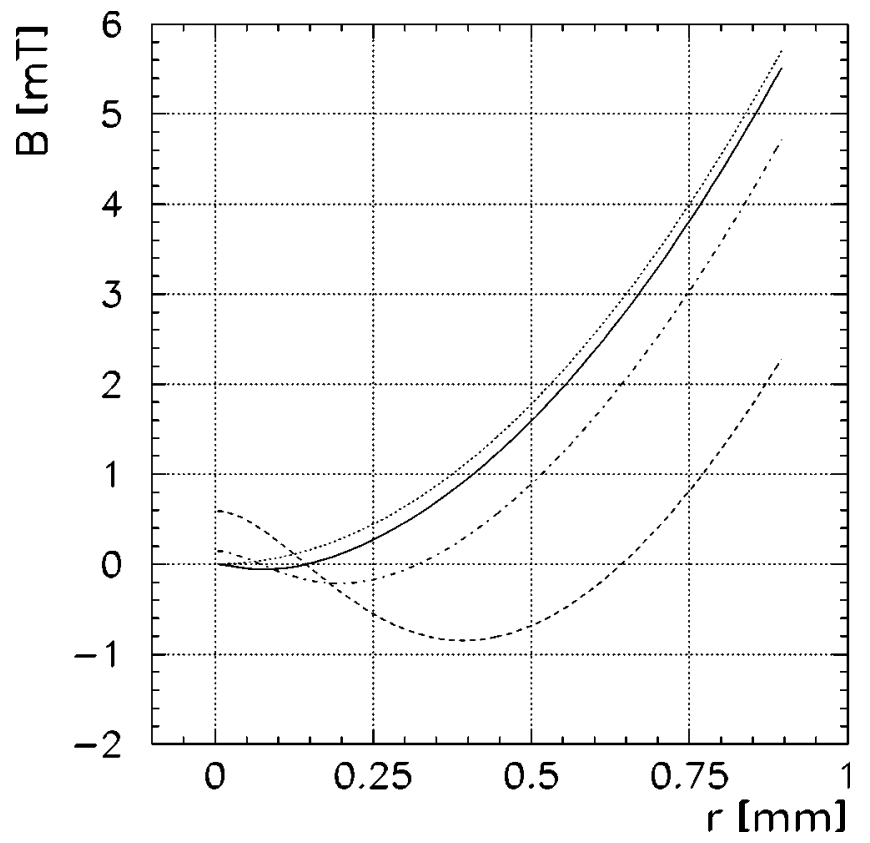

FIG. 15. Radial dependence of the magnetic flux density near the axis of a magnet with $B_{0}=1.6 \mathrm{~T}$ and $r_{1}=15 \mathrm{~mm}$ for three different Hall probe sizes. The dotted line corresponds to a point-like Hall probe, the solid line to a Hall probe of $h=0.2 \mathrm{~mm}$ lateral dimension, the dashed-dotted line to $h$ $=0.5 \mathrm{~mm}$, and the dashed line to $h=1.0 \mathrm{~mm}$.

with a Hall probe of inappropriate dimensions. When higher orders other than $\nu=0$ are omitted, the maximum of the magnetic flux density given in Eq. (A4) simplifies to

$$
\begin{aligned}
B_{\text {meas }}(r)= & B_{0} \cdot\left[8\left(\frac{2 r}{h}\right) \arctan \left(\frac{h}{2 r}\right)-7+\frac{1}{3}\left(\frac{h}{2 r}\right)^{2}\right] \\
& \cdot\left(\frac{r}{r_{1}}\right)^{2},
\end{aligned}
$$

where $B_{0}$ is the quadratic coefficient of the pure sextupole component. From Eq. (A6) it is possible to deduce the Hall probe size $h$ required for measurements near the magnet center. Figure 15 shows the radial dependence of the measured field value $B_{\text {meas }}(r)$ for the three different Hall probes. Obviously, with a Hall probe of finite size a magnetic flux density of zero is measured at two radii already a few tenths of a mm away from the magnet axis, depending on the size of the Hall probe, while in the center the magnetic flux density does not vanish. For the example discussed above $(h=0.2$ $\mathrm{mm}, B_{0}=1.6 \mathrm{~T}$, and $r_{1}=15 \mathrm{~mm}$ ) the limit of

$$
\lim _{r \rightarrow 0} B_{\text {meas }}(r)=\frac{1}{12} B_{0}\left(\frac{h}{r_{1}}\right)^{2}
$$

corresponds to a negligibly small flux density of $B_{\text {meas }}(r$ $\rightarrow 0)=2.4 \times 10^{-2} \mathrm{mT}$.

\section{APPENDIX B: HALL PROBE OFFSET CORRECTION}

In order to account for a transverse displacement of the axis of rotation of the Hall probe with respect to the magnet axis, a correction to the Halbach [Eqs. (6) and (7)] has to be applied. The mathematical derivation leading to Eqs. (10) and (11) is presented in this Appendix.

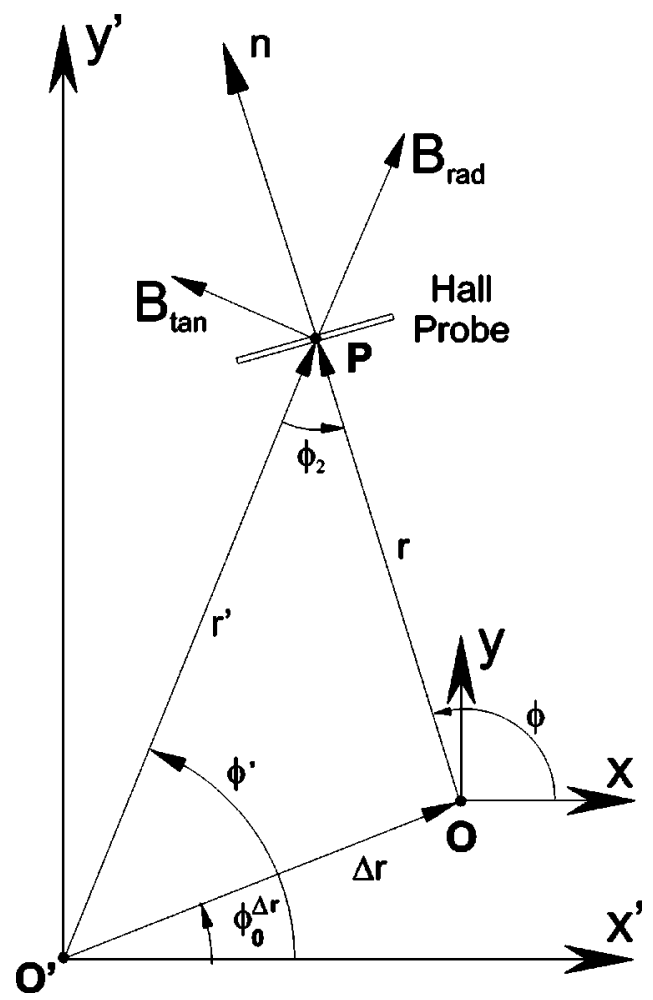

FIG. 16. Coordinate system for azimuthal field measurements. The Hall probe is located at point $P$. The magnet is centered in the $x^{\prime} y^{\prime}$ coordinate system, while the probe is rotated about the center of the $x y$ coordinate system. The distance $\Delta r\left(\ll r, r_{1}\right)$ denotes the offset between magnet axis and axis of rotation on the lathe, $\phi_{0}^{\Delta r}$ corresponds to a starting phase.

The magnet is centered in the $x^{\prime} y^{\prime}$ coordinate system, shown in Fig. 16. Azimuthal measurements are carried out by rotating the Hall probe, located at point $P$, about the center of the unprimed coordinate system, which is displaced with respect to the primed system by $\Delta \vec{r}=(\Delta x, \Delta y)$ $=\left(\cos \phi_{0}^{\Delta r} \Delta r, \sin \phi_{0}^{\Delta r} \Delta r\right)$. The Hall probe responds to the normal projection of the magnetic flux density, thus with the conventions of Fig. 16 one can write

$$
\begin{aligned}
B_{\text {norm }}\left(r, \phi, \Delta r, \phi_{0}^{\Delta r}\right)= & B_{\text {rad }}\left(r^{\prime}, \phi^{\prime}\right) \cos \phi_{2} \\
& +B_{\tan }\left(r^{\prime}, \phi^{\prime}\right) \sin \phi_{2},
\end{aligned}
$$

where $B_{\text {rad }}$ and $B_{\text {tan }}$ are defined by Eqs. (6) and (7). The coordinates $r^{\prime}, \phi^{\prime}$ have to be expressed as functions of $r, \phi, \Delta r$, and $\phi_{0}^{\Delta r}$. Applying the law of sines to the triangle $O O^{\prime} P$ yields

$$
\sin \left(\phi-\phi^{\prime}\right)=\frac{\Delta r}{r} \cdot \sin \left(\phi^{\prime}-\phi_{0}^{\Delta r}\right) .
$$

If one assumes that the offset $\Delta r$ is small compared to $r$, which is justified at larger radii by the alignment precision of the field mapping apparatus, then with $\sin \left(\phi-\phi^{\prime}\right) \approx \phi^{-} \phi^{\prime}$ it follows that

$$
\phi^{\prime}=\phi-\frac{\Delta r}{r} \cdot \frac{\sin \left(\phi-\phi_{0}^{\Delta r}\right)}{1+\frac{\Delta r}{r} \cdot \cos \left(\phi-\phi_{0}^{\Delta r}\right)} .
$$

The law of cosines for the triangle $O O^{\prime} P$ yields a second expression 


$$
r^{\prime 2}=\Delta r^{2}+r^{2}+2 \cdot \Delta r \cdot r \cdot \cos \left(\phi-\phi_{0}^{\Delta r}\right)
$$

Neglecting the quadratic term in $\Delta r$ in the above expression and substituting it in Eq. (B1) the corresponding expressions for $\phi^{\prime}$ and $r^{\prime}$ from Eqs. (B3) and (B4), respectively, yield after some algebraic transformations Eqs. (10) and (11).

${ }^{1}$ For an overview of the different methods applied to polarized beam production, see, e.g., International Workshop on Polarized Sources and Targets, Erlangen, Germany, 1999, edited by A. Gute, S. Lorenz, and E. Steffens (Friedrich-Alexander Universität Erlangen-Nürnberg, 1999); R. J. Holt and M. A. Miller, AIP Conf. Proc. 421 (1998).

${ }^{2}$ N. F. Ramsey, Molecular Beams (Oxford University Press, Oxford, 1956).

${ }^{3}$ J. S. Dunham, C. S. Galovich, H. F. Glavish, S. S. Hanna, D. G. Mavis, and S. W. Wissink, Nucl. Instrum. Methods 219, 46 (1984).

${ }^{4}$ D. Krämer et al., Nucl. Instrum. Methods 220, 123 (1984).

${ }^{5}$ K. Halbach, Nucl. Instrum. Methods 169, 1 (1980).

${ }^{6}$ Sumitomo Special Metals Company Ltd., Egawa, Shimamotocho, Mishimagun, Osaka 618, Japan.

${ }^{7}$ M. Sagawa, S. Fujimura, N. Togawa, H. Yamamoto, and Y. Matsuura, J. Appl. Phys. 55, 2083 (1984).

${ }^{8}$ Y. Wakuta, H. Hasuyama, S. Ikenaga, and K. Yamada, Nucl. Instrum. Methods Phys. Res. A 260, 543 (1987).
${ }^{9}$ Y. Wakuta, K. Maehata, H. Arima, T. Hirose, F. Noda, N. Hichida, and J. Sumita, Rev. Sci. Instrum. 65, 1408 (1998).

${ }^{10}$ R. Hertenberger, Y. Eisermann, A. Hofmann, A. Metz, P. Schiemenz, S. Trieb, and G. Graw, Rev. Sci. Instrum. 69, 750 (1998).

${ }^{11}$ P. Schiemenz, A. Ross, and G. Graw, Nucl. Instrum. Methods Phys. Res. A 305, 15 (1991).

${ }^{12}$ T. Wise, A. D. Roberts, and W. Haeberli, Nucl. Instrum. Methods Phys. Res. A 336, 410 (1993).

${ }^{13}$ F. Stock et al., Nucl. Instrum. Methods Phys. Res. A 343, 334 (1994).

${ }^{14}$ For a listing of materials and magnetic properties, see Table I and RareEarth Permanent Magnets, VACODYM, VACOMAX, Company Information Booklet PD-002, Vacuumschmelze GmbH, P.O.B. 2253, $63412 \mathrm{Ha}-$ nau, Germany, p. 12.

${ }^{15}$ L. G. Isaeva et al., R. J. Holt, and M. A. Miller, AIP Conf. Proc. 421, 109 (1998).

${ }^{16}$ D. Krischel, M. Schillo, A. Hobl, H. Grünberg, and H.-J. Hergesell, Rev. Sci. Instrum. 69, 677 (1998).

${ }^{17}$ ANKE-Collaboration, Apparatus for Nucleon and Kaon Ejectiles, see homepage at http://ikpd15.ikp.kfa-juelich.de:8085/doc/Anke.html

${ }^{18}$ R. Maier, Nucl. Instrum. Methods Phys. Res. A 390, 1 (1997).

${ }^{19}$ MAFIA, CST GmbH, Buedinger Str. 2a, D-64289 Darmstadt, Germany, http://www.cst.de

${ }^{20}$ Keithley Digital Multimeter 2001, $6 \frac{1}{2}$-digit. 